\title{
ANEURYSMAL BONE CYST OF THE CLIVUS- A RARE OCCURRENCE
}

\author{
Sneha Suresh'1, Geetha Devadas ${ }^{2}$
}

${ }_{1}^{1}$ Postgraduate Student, Department of Pathology, Institute of Pathology, Madras Medical College, Chennai.

2 Professor, Department of Pathology, Institute of Pathology, Madras Medical College, Chennai.

ABSTRACT
BACKGROUND
Aneurysmal Bone Cyst (ABC) is a benign lesion, well documented in the metaphysis of the long bones and also in the thorax, pelvis,
and vertebrae. But the occurrence of aneurysmal bone cyst in clivus is rare and accounts for only $1 \%$ of all cases. In this case
report, we hereby discuss about an 11-year-old female who presented with neck swelling for a prolonged duration and
histopathological examination revealed an aneurysmal bone cyst. This case is being presented for its rarity and also to bear in mind
as a differential diagnosis in spite of its rare location.

\section{KEYWORDS}

Aneurysmal Bone Cyst, Benign, Clivus, Rare.

HOW TO CITE THIS ARTICLE: Suresh S, Devadas G. Aneurysmal bone cyst of the clivus- a rare occurrence. J. Evolution Med. Dent. Sci. 2017;6(30):2481-2483, DOI: 10.14260/Jemds/2017/536

\section{BACKGROUND}

Aneurysmal Bone Cysts (ABC) are benign cystic lesions of the bone. They most commonly occur in first two decades of life, having a predilection for the metaphysis of the long bones such as tibia, femur, humerus, posterior elements of vertebral bodies. Aneurysmal bone cyst occurring in the base of skull, particularly clivus is a rare occurrence and accounts for less than or equal to $1 \%$ of the cases. We hereby present a case of an 11-year-old female child who presented with the same.

\section{CASE REPORT}

An 11-year-old female child came with complaints of swelling in the neck for seven years along with restriction of neck movement.

MRI revealed a permeative lytic destruction of right lower half of the clivus along with petrous and squamous part of temporal bone, anterior and lateral mass of the atlas vertebra. An impression of aneurysmal bone cyst was given.

Grossly, we received two grey brown soft tissue fragments measuring $1 \times 1 \times 1 \mathrm{~cm}$ each. The whole tissue was processed.

Histopathology revealed multiple blood-filled cystic spaces with adjoining cellular spindle shaped fibroblastic proliferation interspersed by multinucleate giant cells, hemosiderin laden macrophages. Cystic spaces showed no discernible endothelial lining. With this the diagnosis of aneurysmal bone cyst was given.

Financial or Other, Competing Interest: None.

Submission 01-03-2017, Peer Review 01-04-2017,

Acceptance 07-04-2017, Published 13-04-2017.

Corresponding Author:

Dr. Sneha Suresh

\#2B, Kalyan Apartments,

Abiramapuram, Subramaniam Street,

Chennai-600018.

E-mail: sneha312@gmail.com

DOI: $10.14260 /$ jemds $/ 2017 / 536$

\section{(c) $(1) \ominus$}

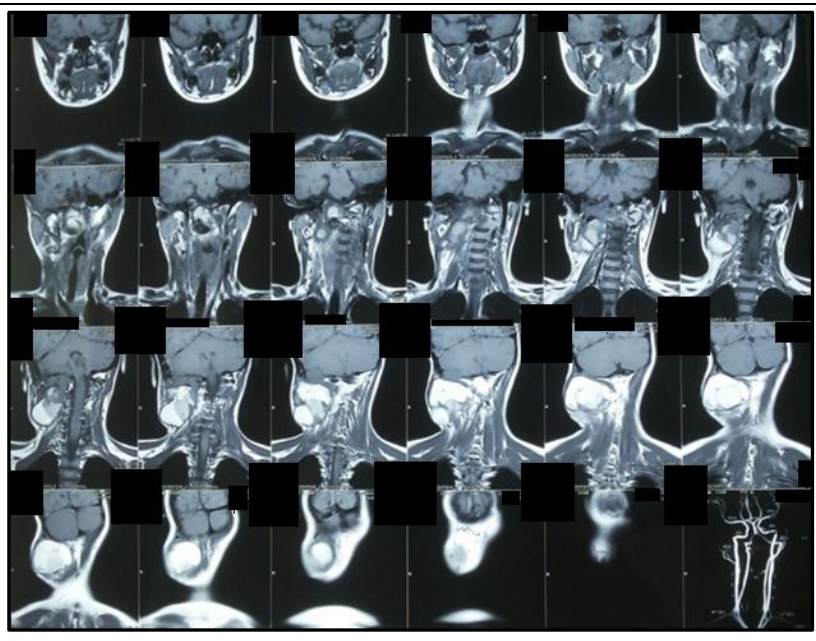

Figure 1. Preoperative MRI-showing a permeative destructive lesion involving lower right half of clivus, petrous and squamous portions of right temporal bone, anterior arch and lateral part of atlas, $\mathrm{C} 3$ and C4 right lateral transverse process. No invasion or thrombi seen. Aneurysmal Bone Cyst of Clivus.

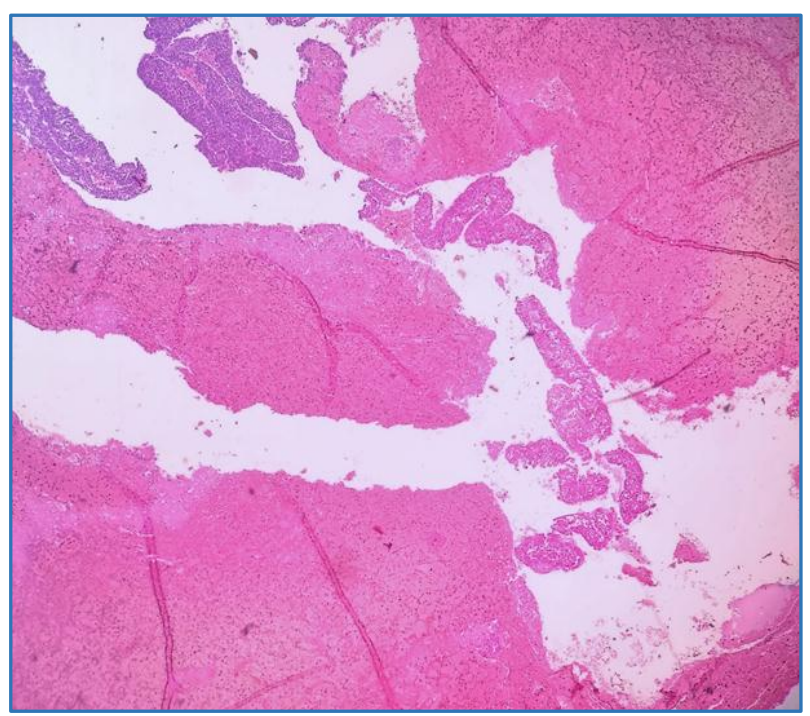

Figure 2. Showing a lesion with dilated vascular spaces separated by fibrous stroma. (40x magnification) 


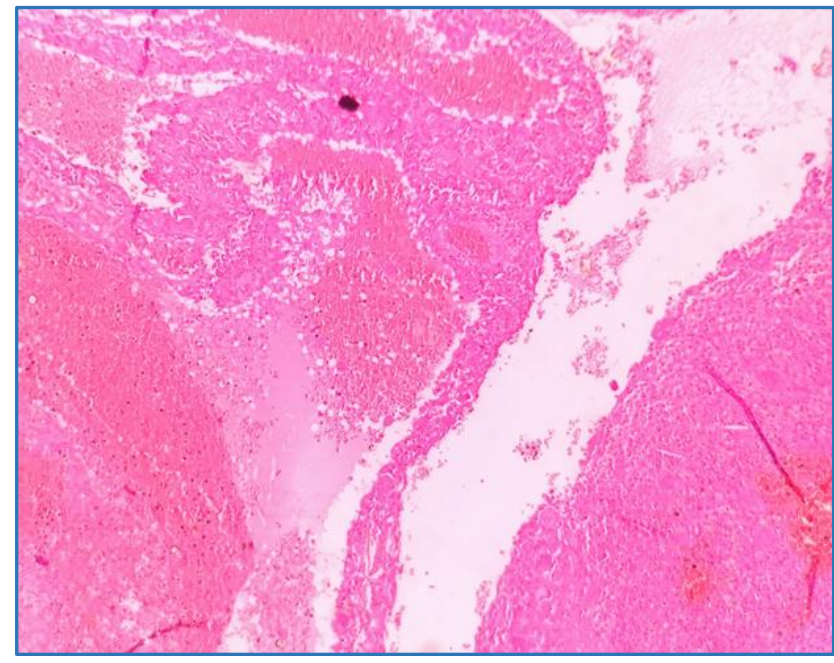

Figure 3. Shows vascular spaces not lined by any discernible endothelial lining. (100x magnification).

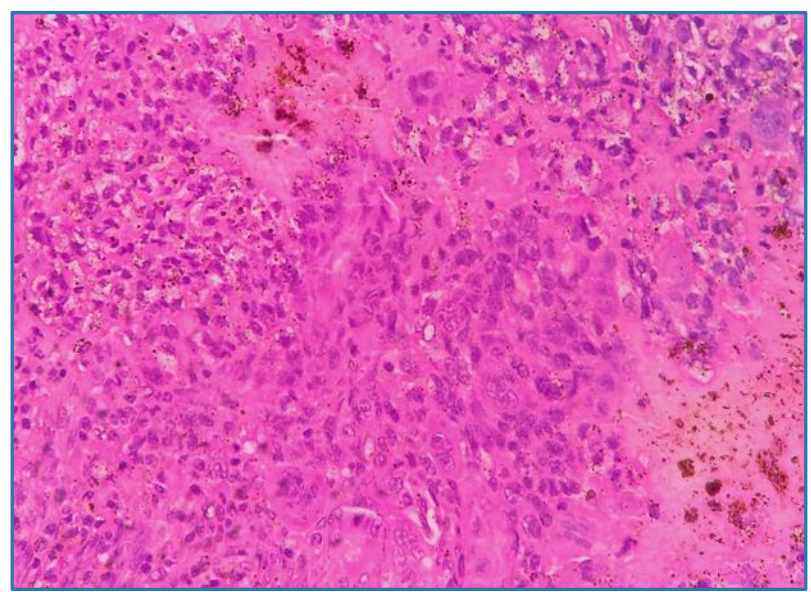

Figure 4. Shows fibrous stroma with numerous hemosiderin laden macrophages. (400x magnification).

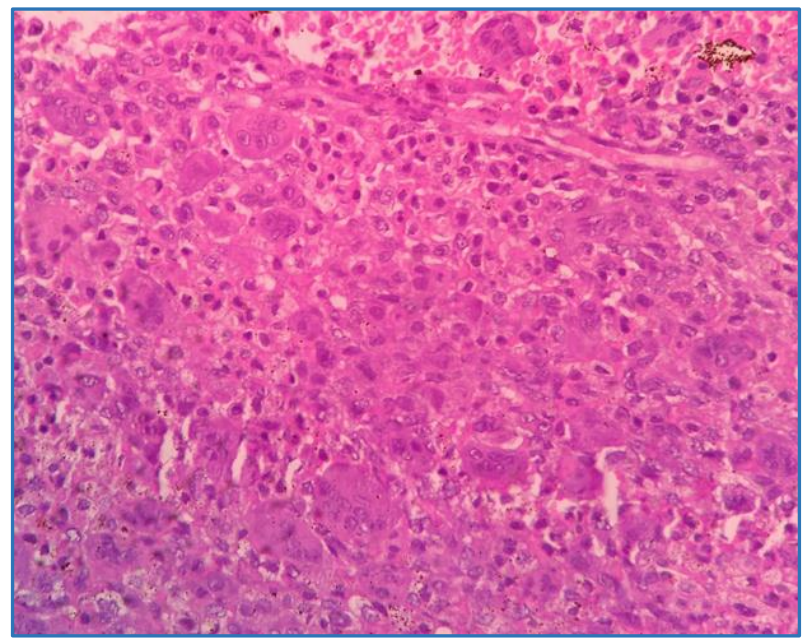

Figure 5. Fibrous stroma shows numerous multinucleated giant cells

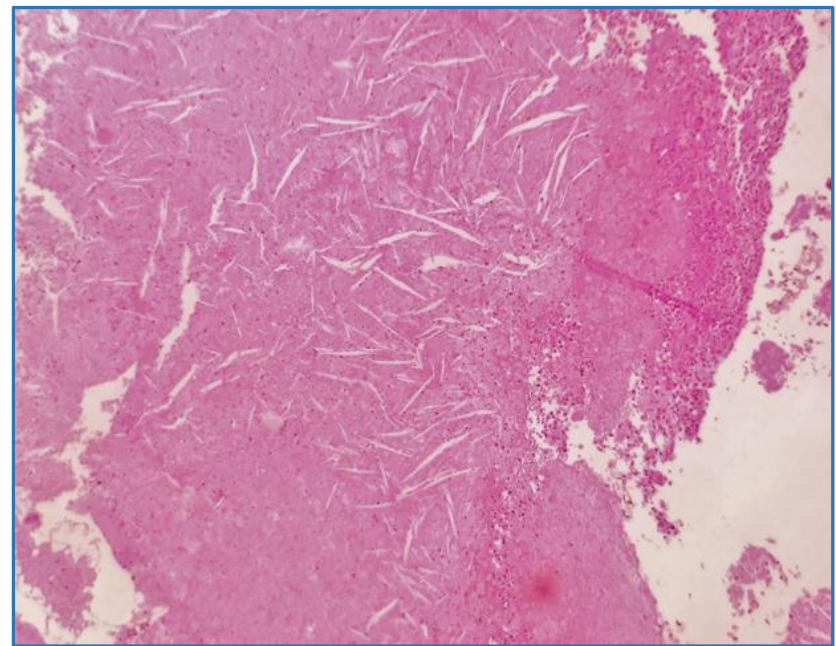

Figure 6. Shows areas of cholesterol clefts. (100x magnification).

\section{DISCUSSION}

Aneurysmal Bone Cyst is a benign, well-circumscribed, lytic, expansile lesion of the bone that can be locally destructive. ${ }^{1}$ This expansile bone lesion contains blood-filled cystic cavities. Occurrence in the metaphysis of long bones is common. Other sites of involvement are flat bones and the vertebral column. 25\% involve the vertebra, body, hyoid, mandible and odontoid. As in our case the first two decades are more commonly affected. In some cases, diagnosis of Aneurysmal Bone Cyst is difficult because of overlapping radiologic and pathologic features with other benign and malignant tumours, especially when it occurs in an uncommon site, like the base of the skull. Clinical presentation of Aneurysmal Bone Cyst depends on the site of involvement.

Aneurysmal Bone Cyst of skull base is relatively rare and constitutes only $1 \%$ of cases. ${ }^{2,3}$ Review of literature reveals that by far only 11 cases of $\mathrm{ABC}$ of clivus have been reported. ${ }^{3}$

There are three phases in the development of aneurysmal bone cyst, namely 1 . Initial phase, with predominantly osteolysis. 2. Growth phase, with rapid increase of the tumour, expansion of bone, and marked bone destruction. The tumour is not demarcated, and septae are indistinct. Gradually, the first signs of a bony shell appear around the tumour. 3. Stabilisation phase, with fully developed radiologic syndrome.

World Health Organisation (WHO) classifies Aneurysmal Bone Cyst in the category of tumours of undefined neoplastic nature and the exact pathogenesis of this disease is not known. Postulates like alteration of local blood flow, secondary trauma have been suggested. $3,4,5,6$

There are two forms of ABC- Primary (Occurring de novo) and secondary which complicates other benign and malignant conditions like-Fibrous Dysplasia, Osteoblastoma, Osteosarcoma, Chondroblastoma, Giant Cell tumour, chondromyxoid fibroma, non-ossifying fibroma.4,5,6,7 In our case all the causes of secondary $A B C$ were ruled out. Histologically it is a well-circumscribed lesion composed of blood-filled cystic spaces separated by fibrous septa. 
Septae are composed of fibroblasts, multinucleated giant cells. The blood-filled cavities are characteristically not lined by endothelium. In case of Secondary ABC, the longstanding lesion is identified in addition to features of $\mathrm{ABC}$.

The Differential Diagnoses Considered were as follows. Giant Cell Tumour (GCT)

Due to the paucity of giant cells in comparison to a GCT and the unequal distribution of giant cells and the lack of similarity between the Giant cell nuclei and stromal cells, GCT was ruled out.

\section{Eosinophilic Granuloma}

Has a predilection for the skull bones. Langerhans cells were absent. Immunohistochemistry performed with CD1a was negative.

\section{Chordoma}

Was considered in view of clival location; however, the characteristic large physaliferous cells were not evident and we ruled out the same.

\section{CONCLUSION}

$\mathrm{ABC}$ of clivus is a rapidly growing expansile lesion and requires immediate attention. ${ }^{7}$ The unusual site of occurrence should be borne in mind due to proximity to the vital base of skull structures thereby endangering the patient's life in spite of being a benign lesion. This case is presented for its rarity.

\section{REFERENCES}

[1] Ustabasioglu FE, Samanci C, Asik M, et al. Aneurysmal bone cyst of sphenoid bone and clivus misdiagnosed as chordoma: a case report. Brain Tumor Res Treat 2015;3(2):115-7.

[2] Aghaghazvini L, Sedighi N, Karami P, et al. Skull base aneurysmal bone cyst presented with foram en jugular syndrome and multi-osseous involvement. Iran J Radiol 2012;9(3):157-60.

[3] Hnenny L, Roundy N, Zherebitskiy V, et al. Giant aneurysmal bone cyst of the anterior cranial fossa and paranasal sinuses presenting in pregnancy: case report and literature review. J Neurol Surg Rep 2015;76(2):e216-21.

[4] Rajput D, Tungaria A, Jaiswal A, et al. Aneurysmal bone cyst of clivus and C1 C2: case report and review of literature. Turk Neurosurg 2012;22(1):105-8.

[5] Kalina P, Wetjen N. Aneurysmal bone cyst of the occipital bone. J Pediatr 2015;167(2):496-e2.

[6] Bozbuğa M, Süslü TH. Aneurysmal bone cyst of the sphenoid bone extending into the ethmoid sinus, nasal cavity and orbit in a child. Turk Neurosurg 2009;19(2):172-6.

[7] Ruiter DJ, van Rijssel TG, van der Velde EA. Aneurysmal bone cysts: a clinicopathological study of 105 cases. Cancer 1977;39(5):2231-9. 